\title{
Interpreting Graphic Models
}

\author{
J. C. Nordbotten \\ Dept. of Information Science, University of Bergen, \\ N-5020 Bergen, Norway (e-mail joan@ifi.uib.no) \\ M. E. Crosby \\ Dept. of Information and Computer Science, \\ University of Hawaii at Manoa, \\ Honolulu, HI 96822, USA (e-mail crosby@uhics.ics.hawaii.edu)
}

When using graphic models for system specification and documentation, it is important that the information presented is readily comprehensible to the intended readers. For data models, the readers are primarily the system users, who are asked to confirm the correctness of the information and data requirements modelled, and the system implementers who are to implement the database system in accordance with the specifications given in the model.

It is commonly argued that graphic models enhance understanding. The authors of graphic data models appear to assume that the graphic model is a sufficient specification tool, in as much as it is seldom supplemented with a definition language other than translation rules to a relational model. We have studied user comprehension of 3 different representation styles (1) used for graphic data models. Our measure of user comprehension is the percentage of correctly identified model components.

Our study indicates that the graphic representation used for a model influences the quality of interpretation. Interpretation scores for the highly graphic models in our study were significantly (2) lower than the interpretation scores for minimally graphic models or for models using an embedded symbol structure. An explanation could be graphic overload which hinders component identification.

Further, our observations show that graphic models are not completely read. Up to 3010-20models alone are not dependable tools for either requirements confirmation or reliable system implementation.

It has been assumed that graphic models would be read in a text-like serial manner. However, the most proficient interpreters focused on identification and presentation of the model structure, given in the central portion of the models. All subjects used this interpretation strategy for the familiar model types, indicating that it can be learned.

$\begin{array}{lll}\text { (1) Graphic styles: } & \text { Highly graphic, } & \text { eg. NIAM [Nijssen, G.M. '89], } \\ & \text { Embedded, } & \text { eg. OODM [Cattel, R.G.G.'91], } \\ \text { and } & \text { Minimally graphic, } & \text { eg. SSM [Nordbotten '93]. }\end{array}$

(2) $\mathrm{p}=0.049$ and $\mathrm{p}=0.038$, using a paired t-test, for the NIAM : OODM and NIAM : SSM model interpretations respectively. 